\title{
Introduction to the JINS Special Issue: Neurodevelopmental Disorders
}

\author{
E. Mark Mahone, ${ }^{1,2}$ Seth Warschausky, ${ }^{3}$ AND T. Andrew Zabel ${ }^{1,2}$ \\ ${ }^{1}$ Kennedy Krieger Institute, Baltimore, Maryland \\ ${ }^{2}$ Johns Hopkins University School of Medicine, Baltimore, Maryland \\ ${ }^{3}$ University of Michigan, Ann Arbor, Michigan
}

Neurodevelopmental disorders are conditions that involve early insult or abnormality in the developing central nervous system and are associated with a wide spectrum of abilities. These conditions begin during the early developmental period (usually conceptualized as prenatally though the first 3 years of life), affect day-to-day functioning, and are often lifelong. Because the "typical" development of the nervous system has been altered in individuals with neurodevelopmental disorders, reorganization and competition for function occur, usually resulting in skill patterns that are less efficient than among individuals without such conditions. The timing of these alterations or developmental disruptions is also relevant, as different neural systems are selectively vulnerable to injury at different phases of prenatal and post-natal development. As a result, the behavioral and cognitive dysfunction associated with early neural damage can range from subtle (or absent) to diffuse and profound. Moreover, the functional impairments can be observed immediately in some individuals, while in others, the full range of deficits may not manifest until later in life, even though the neurobiological basis of the condition is present earlier (Rudel, 1981).

Among children with neurodevelopmental disorders, the trajectory is often "off developmental track" relative to the trajectory of typically developing children. Developmental delays (i.e., patterns of skill development that should have occurred earlier in life) are often observed early in life. While functional catch-up is possible, it is often incomplete, and the resulting maturational timelines based on typical development become less applicable (Mahone, Slomine, \& Zabel, 2018).

Neurodevelopmental disorders are highly prevalent. Recent estimates from the Centers for Disease Control and Prevention (CDC) in the United States show that around one in six, or approximately $17 \%$, of children ages 3 through 17 years have one or more neurodevelopmental disabilities (Boyle et al., 2011). The rates also are increasing, and the CDC reports may underestimate the actual prevalence worldwide. In the past 25 years, medical advances have improved the life course of several genetic, medical, and neurodevelopmental conditions, making them more survivable and compatible with life (e.g., very low birth weight preterm infants, congenital hydrocephalus) and extending the expected lifespan of others (e.g., cystic fibrosis, sickle cell disease). Due to higher survival rates and lifespans extending into adulthood, increased attention has been given to the development of selfmanagement and independence skills and the transition into older adolescence and young adulthood (Tarazi, Mahone, \& Zabel, 2007; Warschausky, Kaufman, Evitts, Schutt, \& Hurvitz, 2017; Zabel, Jacobson, \& Mahone, 2013). Given these considerations, the assessment and study of individuals with neurodevelopmental disorders is of significant interest to neuropsychologists.

Classification of neurodevelopmental disorders can be conceptualized using two primary approaches, one emphasizing behavior (without explicit reference to etiology), and the other emphasizing etiological medical, genetic, and neurological factors (Mahone et al., 2018). In the field of neuropsychology, those neurodevelopmental disorders defined on the basis of behavior (including attention-deficit/hyperactivity Disorder, ADHD; learning disabilities, LDs; autism spectrum disorders, ASDs; and intellectual disability, ID) have received considerable emphasis, in part because of their prevalence and overall public health relevance (Leigh \& Du, 2015; Mahone \& Denckla, 2017; Mahone \& Mapou, 2014). Neurodevelopmental disorders diagnosed on the basis of known or presumed medical etiologic factors have received somewhat less emphasis among neuropsychologists. Such conditions include those with genetic, environmental (injury, infection, teratogens), or multi-factorial medical etiologies.

This special issue of the Journal of the International Neuropsychological Society focuses upon such conditions with known medical or genetic etiologies, and includes 11 papers presenting innovative and novel data related to the neuropsychology (including identification of biomarkers) of specific neurodevelopmental disorders. Included in the issue are seven studies reporting new empirical findings, two critical reviews, and two case reports. The timing of this special issue follows on the heels of the 50th anniversary of the implementation of US PL-88-164 ("Mental Retardation Facilities Construction Act"), which, in 1967, provided 
financial support for the development 18 University Affiliated Programs (emphasizing treatment for neurodevelopmental disorders), and 12 Research Centers dedicated to research of neurodevelopmental disorders, all of which have contributed to the scientific innovations that have improved the lives of individuals with neurodevelopmental disorders and their families.

The issue begins with seven empirical studies, emphasizing disorders (both rare and more common) with genetic and associated medical etiologies, with samples ranging in age from early childhood through young adult. Williams syndrome is a rare genetic condition, often associated with intellectual disability and significant visuospatial dysfunction. In the first paper, Prieto-Corona and colleagues report on neuropsychological and functional outcomes in children with Williams syndrome, with and without the additional (even rarer) deletion of the GTF2IRD2 gene. They showed that those individuals with the additional genetic deletion had even greater dysfunction in visuospatial and social cognition, compared to those with without the deletion.

Antschel et al. report findings from a rich, 9-year longitudinal dataset of individuals with 22q11.2 deletion syndrome, a disorder associated with high risk for functional impairment and psychosis. They found that early executive function, especially working memory deficits, were associated with later functional impairment, but that the association was seen in both those with and without the disorder, highlighting the importance of early assessment of executive and cognitive control skills as predictors of later outcome.

There is considerable sexual dimorphism observed among individuals with neurodevelopmental disorders. The study of individuals with sex chromosome aneuploidies-conditions characterized by abnormal numbers of X or Y chromosomes, for example, Klinefelter syndrome (XXY) or Turner syndrome (XO) - provides a highly relevant framework to investigate the etiology of some sex differences in development and function. In this issue, Udhnani and colleagues and Maiman and colleagues report on a less studied variant of sex chromosome aneuploidies-those with trisomies, tetrasomies, and pentasomies-showing an association between these variants and reductions in verbal fluency, with severity of deficits related linearly to the number of supernumerary $\mathrm{X}$ chromosomes.

The dystrophinopathies (including Duchenne and Becker muscular dystrophies) are $\mathrm{X}$-linked muscle diseases associated with abnormal expression of the protein dystrophin. These conditions affect primarily males and result in a wide range of functional cognitive deficits. Fee and colleagues report on neuropsychological performance in a sample of 50 boys with muscular dystrophy, grouped by gene mutation position relative to exon 43 . They found that boys with mutation downstream from exon 43 showed greater academic deficits, relative to those with mutation upstream of exon 43.

Medical and surgical advances contribute to an increasing number of individuals surviving congenital heart disease (CHD) and its treatment. King et al. report on neuroimaging findings in a sample of adolescents and young adults with
CHD, showing reduced cerebellar volumes, with reductions predictive of executive and cognitive control functions.

The manifestation of neurobehavioral dysfunction among children with neurodevelopmental disorders often occurs early in life. Downes and colleagues present a case control study of executive functions in preschoolers with sickle cell disease (SCD). In their sample, performance-based reductions in inhibitory control and cognitive flexibility were more pronounced than parent reports of similar functions, highlighting the importance of direct assessment of executive control skills in preschoolers with SCD.

Down syndrome (DS) represents the most common genetic etiology of intellectual disability, and is associated with a wide range of medical complications and skill difficulties, especially those implicating hippocampally mediated functions. Edgin and colleagues reported minimal effects of a fast-mapping strategy, hypothesized to incrementally improve word retention, but instead showed that individuals with DS do retain novel words effectively, but only when presented during learning trials in small groups. In a related review, Hammer and colleagues provide a succinct overview of structural anatomic neuroimaging studies of individuals with DS, highlighting widespread reductions in cerebral volume early in life, with smaller effects (relative reductions) observed by adolescence.

Neurofibromatosis type 1 (NF1) is a genetic neurocutaneous disorder associated with learning disabilities, ADHD, and an increased risk for brain tumors. Beaussart and colleagues provide a meta-analysis of 19 studies of individuals with NF1, emphasizing executive control skills. They concluded that, in general, working memory and planning skills were relatively more affected than inhibitory control in this population, and that relative difficulties (compared to those without NF1) tend to increase with age through adolescence.

The two final papers in this issue highlight the utility of case studies, especially in rare conditions. Tan et al. report on an individual with Pitt-Hopkins syndrome (PHS), a rare genetic disorder caused by insufficient expression of the TCF4 gene. Nearly all of the few prior published reports on PHS highlight severe intellectual and functional deficits and minimal language use. This case report instead presents findings from an individual who, despite many cognitive limitations, showed some relatively spared language function. In the final paper for this special issue, Kim et al. report on an intervention using different spacing methods to improve word list learning in a young adult with congenital amnesia secondary to premature birth and associated hypoxic-ischemic injury. They found that word recognition improved with repetitions spaced, rather than massed.

As illustrated in this set of papers, neuropsychological studies of neurodevelopmental disorders typically are conducted from a developmental perspective with an increasingly interdisciplinary approach that frequently draws upon (and informs) a refined understanding of endophenotypes and biomarkers. The ultimate hope, of course, is that these research approaches will inform more effective treatment and optimal developmental outcomes for the target populations. 
It was a pleasure organizing these papers into this special issue, and we thank the authors for their contribution to this unique collection of studies demonstrating the importance of rigorous neuropsychological inquiry into neurodevelopmental conditions. It is our hope that the readers of the Journal of the International Neuropsychological Society find this collection valuable and are able to build off of the innovative and novel neuropsychological findings in the specific neurodevelopmental disorders presented within.

\section{REFERENCES}

Boyle, C.A., Boulet, S., Schieve, L., Cohen, R.A., Blumberg, S.J., Yeargin-Allsopp, M., ... Kogan, M.D. (2011). Trends in the prevalence of developmental disabilities in US children, 19972008. Pediatrics, 27, 1034-1042. doi:10.1542/peds.2010-2989

Leigh, J.P., \& Du, J. (2015). Brief report: Forecasting the economic burden of autism in 2015 and 2025 in the United States. Journal of Autism and Developmental Disorders, 45, 4135-4139. doi:10.1007/s10803-015-2521-7

Mahone, E.M., \& Denckla, M.B. (2017). Attention-deficit/ hyperactivity disorder: A historical neuropsychological perspective. Journal of the International Neuropsychological Society, 23 , 916-929. doi:10.1017/S1355617717000807
Mahone, E.M., \& Mapou, R. (2014). Learning disabilities. In K. Stucky, M. Kirkwood, J. Donders, \& C. Liff (Eds.). Clinical neuropsychology study guide and board review (pp. 184-201). New York, NY: Oxford University Press.

Mahone, E.M., Slomine, B.S., \& Zabel, T.A. (2018). Genetic and neurodevelopmental disorders. In J. Morgan \& J. Ricker (Eds.) Textbook of Clinical Neuropsychology (2nd ed., pp. 127-140). New York: Psychology Press.

Rudel, R.G. (1981). Residual effects of childhood reading disabilities. Annals of Dyslexia, 31, 89-102.

Tarazi, R., Mahone, E.M., \& Zabel, T.A. (2007). Self-care independence in children with neurological disorders: An interactional model of adaptive demands and executive dysfunction. Rehabilitation Psychology, 52, 196-205. doi:10.1037/00905550.52.2.196

Warschausky, S., Kaufman, J., Evitts, M., Schutt, W., \& Hurvitz, E.A. (2017). Mastery motivation and executive functions as predictors of adaptive behavior in adolescents and young adults with cerebral palsy or myelomeningocele. Rehabilitation Psychology, 62, 258-267. doi:10.1037/rep 0000151

Zabel, T.A., Jacobson, L., \& Mahone, E.M. (2013). Hydrocephalus/ spina bifida. In I.S. Baron, \& C. Rey-Casserly (Eds.), Pediatric neuropsychology: medical advances and lifespan outcomes (pp. 279-301). New York, NY: Oxford University Press. 